\title{
Silent Positioning in Underwater Acoustic Sensor Networks
}

\author{
Xiuzhen Cheng \\ Computer Science \\ The George Washington University \\ Washington, DC 20052, USA \\ cheng@gwu . edu
}

\author{
Haining Shu \& Qilian Liang \\ Electrical Engineering \\ University of Texas at Arlington \\ Arlington, TX 76019, USA \\ \{shu@wcn.uta.edu, liang@uta.edu\}
}

\author{
David Hung-Chang Du \\ Computer Science \& Engineering \\ University of Minnesota \\ Minneapolis, MN 55455, USA \\ duecs. umn . edu
}

August 16, 2007

\begin{abstract}
In this paper, we present a silent positioning scheme termed as UPS for underwater acoustic sensor networks. UPS relies on the time-difference of arrivals measured locally at a sensor to detect range differences from the sensor to four anchor nodes. These range differences are averaged over multiple beacon intervals before they are combined to estimate the 3D sensor location through trilateration. UPS requires no time-synchronization and provides location privacy at underwater vehicles/sensors whose locations need to be determined. To study the performance of UPS, we model the underwater acoustic channel as a modified Ultra Wide Band (UWB) S-V model: the arrival of each path cluster and paths within each cluster follow double Poisson distributions, and the multipath channel gain follows a Rician distribution. Based on this channel model, we perform both theoretical analysis and simulation study on the position error of UPS under acoustic fading channels. The obtained results indicate that UPS is an effective scheme for underwater vehicle/sensor self-positioning.
\end{abstract}

Keywords: Underwater acoustic sensor networks, localization, navigation, UWB S-V model, underwater GPS, underwater positioning. 


\section{Introduction}

UnderWater Acoustic Sensor Networks (UWA-SNs) consists of a variable number of sensors and vehicles (Unmaned Underwater Vehicle (UUV), Autonomous Underwater Vehicle (AUV), etc.) to perform collaborative monitoring tasks over a given area. The main motivation for UWA-SNs is their relative ease of deployment since they eliminate the need for cables and they do not interfere with shipping activities. UWA-SNs are envisioned to enable applications for environmental monitoring of physical and chemical/biological indicators, tactical surveillance, disaster prevention, undersea exploration, assisted navigation, etc.

Location discovery for underwater vehicles/sensors is nontrivial in the oceanic medium. Propagation delays, motion-induced Doppler shift, phase and amplitude fluctuations, multipath interference, etc., are all significant factors in location measurement. The well-known Global Positioning System (GPS) receivers, which may be used in terrestrial systems to accurately estimate the geographical locations of sensor nodes, do not work properly in underwater [6]. Some localization schemes based on received signal strength (RSS), time of arrival (ToA), or angle of arrival (AoA), could be used. Nevertheless, the bandwidth constraint, sensor mobility, and unpredicted variation in channel behavior make many of these approaches inaccurate or unapplicable [14]. For example, the Doppler shift introduced by mobility affects the AoA algorithm, and the underwater power loss model (depending on distance and frequency) makes the RSS-based estimation results ambiguous. More over, the accuracy of the localization relates to the bandwidth of the signal and the SNR at the receiver (pp. 429, [4]). The lower limit for $\sigma^{2}$ estimation in the presence of AWGN is given by the Cramer-Rao lower bound, $\sigma^{2}=\frac{N_{0}}{2 \int_{-\infty}^{+\infty}(2 \pi f)^{2}|p(f)|^{2} d f}$, which indicates that $\sigma^{2}$ is inversely proportional to the bandwidth. Unfortunately, the bandwidth of UWA-SNs is significantly limited, which theoretically demonstrates that acoustic positioning in UWA-SNs is very challenging.

Intuitively, ToA or TDoA based localization should be preferable. Nevertheless, ToA or TDoA approaches require time synchronization if one-way sound flying time is counted on; otherwise, a ping-pong style round trip propagation delay needs to be measured. In underwater acoustic sensor networks, precise time synchronization is hard to achieve due to the characteristics of sound when travelling in water [26]. In addition, the low bandwidth of acoustic signals is shared by navigation and data communication in UWA-SNs [24], therefore the ping-pong style alternative may significantly decrease the network throughput. For the same reason, schemes requiring a large number of anchor nodes whose locations are known a priori are prohibitive to UWA-SN. In this paper, we propose UPS, a ToA-based silent Underwater Positioning Scheme, to carefully address the concerns and challenges mentioned above.

The major contribution of this paper lies in three aspects. First, we propose UPS, a silent positioning scheme for UWA-SNs and demonstrate our algorithm by simulation. Second, we investigate the propagation delay and multipath channel. We found that in acoustic underwater networks with large propagation delays, a multipath channel can be modelled as a modified Ultra Wide Band (UWB) Saleh-Valenzuela model (S-V): the arrival of each cluster and the paths within each cluster follow double Poisson distributions and the multipath channel gain follows a Rician distribution. Third, we analyze the theoretical performance of our scheme in propulsion noise environments and identify the possible sources of errors with measures to help mitigate them. Compared to existing schemes proposed in the context of underwater acoustic sensor networks, UPS has the following characteristics and advantages:

- UPS utilizes very few number of anchor nodes (four anchors in our study) and requires no special hardware to provide 3D localization.

- UPS requires no time synchronization. All time differences are computed from the measurements by a local timer.

- UPS provides silent positioning. Underwater vehicles and sensors do not actively transmit any beacon signal. They just passively listen to the broadcastings of the anchor nodes for self-positioning.

- UPS has low computation overhead. It is based on simple algebraic operations on scalar values.

- As evidenced by our simulation study, UPS has low position error. It is applicable to both localization and navigation in UWA-SNs.

The silent positioning feature of UPS deserves further emphasis. First, it can significantly conserve bandwidth and therefore improve network throughput since sensors/vehicles do not transmit any beacon for positioning purpose. 
This is particularly true when a large number of vehicles and sensors need to be positioned in a UWA-SN. Second, UPS is applicable to asymmetric UWA-SNs where the transmission from an underwater vehicle or sensor could not reach four or more anchor nodes. Third, silent positioning provides strong location privacy, which can help to protect sensors/vehicles from being detected in critical applications.

This paper is organized as follows. Section 2 summarizes the major related works in UWA-SNs. Section 3 proposes UPS, a silent underwater positioning scheme for UWA-SN. Underwater acoustic channel modeling and theoretic performance analysis are given in Section 4. Simulation results are reported in Section 5. We conclude our paper and report our future research in Section 6.

\section{Related Work}

Sensor self-positioning has been extensively studied for typical indoor and outdoor sensor networks [15, 19]. In this section, we briefly overview the localization techniques proposed in UWA-SNs. For a more detailed literature survey, we refer the readers to [14] and the references therein.

Underwater acoustic localization can be broadly classified into two categories: range-based and range-free. Range based schemes first measure or estimate distances or angles to a small number of anchor nodes via ToA, RSS, AoA, or even network connectivity, and then apply triangulation or multi-lateration to transform ranges into coordinates. Range-free schemes explore the local topology and the position estimate is derived from the locations of the surrounding anchor nodes. Generally speaking, range-based schemes have higher position accuracy while range-free provides coarser location estimation.

An area-based range-free underwater positioning (ALS) is proposed in [13]. ALS relies on variable power levels of anchor nodes to partition the plan into areas. Each anchor node has its own non-overlapping partition. A vehicle/sensor receives its position estimate from a central server after providing all the areas (one for each anchor node) it resides. UPS is a range-based scheme with much higher position accuracy.

Range-based underwater localization requires either long-range or short-range anchors. Since short-range beacon covers a smaller space, a larger number of anchor nodes are involved and therefore it is unfavorable in underwater environment. Motivated by terrestrial GPS, underwater GPS such as GIB (GPS Intelligent Buoys) [8] and PARADIGM [7] have been proposed. Even though PARADIGM is able to compute location on-board, GIB relies on a centralized server to compute location for underwater vehicles/sensors. These two methods require time-synchronization for ToA measurement between anchor nodes and underwater vehicles/sensors. Hahn and Rice [17] propose a ping-pong style scheme to measure the round-trip delay for range estimation. All these long-range based methods require underwater vehicles/sensors to interrogate with multiple surface buoys, which contributes to network throughput degradation compared to UPS's silent positioning.

If there is no direct communication between anchor nodes and sensors, network connectivity can be explored for range estimation. In [23], three range detection methods based on network connectivity have been proposed: DVhop, DV-distance, and Euclidean. Comparison study in [23] indicates that Euclidean performs the best in anisotropic topologies with a tradeoff of larger computation and communication overheads. Zhou et al. [29] has extended the Euclidean method to 3D UWA-SN and studied its performance. This method relies on a relatively larger number of anchor nodes, which results in higher deployment cost. Zhang and Cheng [28] proposes UR-PLACE, a protocol for underwater robot self-positioning that exploits the multihop connectivity to anchor nodes via beacon flooding. The extensive local communication in [29] and the global flooding in [28] worsen the bandwidth shortage problem in UWA-SN, which unavoidably degrades the network throughput. As a comparison, our UPS requires no active transmission from underwater vehicles/sensors.

\section{UPS: An Underwater Positioning Scheme}

In this section, we propose UPS, a silent acoustic positioning scheme for underwater vehicle/sensor localization. UPS is motivated by our previous work presented for 2D terrestrial sensor networks [15,21,27], which rely on the ToA of RF signals from three anchor nodes for location estimation. The propagation characteristics of RF signals in free space and that of acoustic signals underwater are significantly different, which fundamentally affect the performance of any position algorithm. 
UPS consists of two steps. The first step detects the differences of signal arrival times from four anchor nodes. These time differences are transformed into range differences from the underwater vehicle/sensor to the anchor nodes. In the second step, trilateration is performed to transform these range estimates into coordinates. In the following, we first discuss the network model under our consideration.

\subsection{Network Model}

We assume that an UWA-SN consists of mobile underwater vehicles (e.g. UUVs or AUVs) and stationary sensors. UUVs and AUVs move about at a typical speed of around 2 meters [24] within a confined space, which also covers all non-mobile sensors. To ease our elaboration, from now on we use "sensor" to denote both a mobile vehicle or a stationary sensor. There exist at least four non-cospace anchor nodes with long-range beacons whose locations are known a priori. Each of them is equipped with an acoustic transmitter that can cover the whole activity space. No three anchors are collinear. An example layout of anchor nodes is illustrated in Fig. 1.

\subsection{A Time-Based Location Detection Scheme}

Given the locations $\left(x_{a}, y_{a}, z_{a}\right),\left(x_{b}, y_{b}, z_{b}\right),\left(x_{c}, y_{c}, z_{c}\right)$, and $\left(x_{d}, y_{d}, z_{d}\right)$ of anchor nodes $\mathrm{A}, \mathrm{B}, \mathrm{C}$, and $\mathrm{D}$, respectively, we are going to determine the location $(x, y, z)$ of sensor $S$, as shown in Fig. 1 . Let $d_{i j}$ be the distance between $i$ and $j$, where $i, j \in\{a, b, c, d, s\}$, representing the four anchor nodes and the sensor $S$. We have

$$
\begin{aligned}
d_{a b} & =\sqrt{\left(x_{a}-x_{b}\right)^{2}+\left(y_{a}-y_{b}\right)^{2}+\left(z_{a}-z_{b}\right)^{2}} \\
d_{a c} & =\sqrt{\left(x_{a}-x_{c}\right)^{2}+\left(y_{a}-y_{c}\right)^{2}+\left(z_{a}-z_{c}\right)^{2}} \\
d_{a d} & =\sqrt{\left(x_{a}-x_{d}\right)^{2}+\left(y_{a}-y_{d}\right)^{2}+\left(z_{a}-z_{d}\right)^{2}}
\end{aligned}
$$

The first step of UPS computes the range differences between $d_{s a}$ and $d_{s b}, d_{s c}, d_{s d}$, respectively.

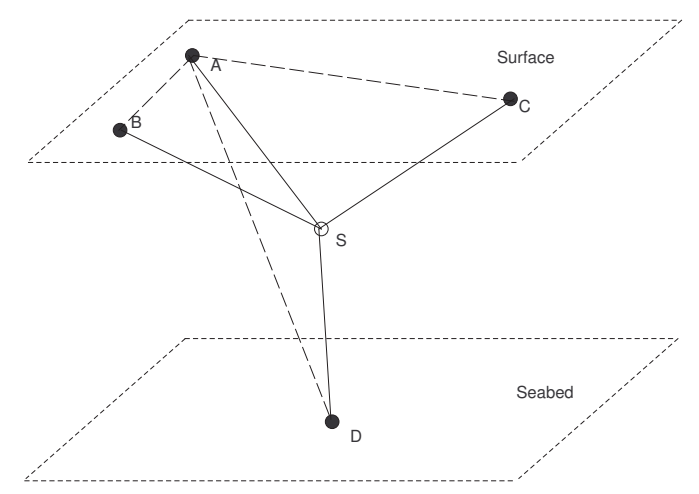

Figure 1: Sensor $S$ will measure the arrival times of beacon signals from anchor nodes A, B, C, and D locally. $S$ also will receive the turn-around delay information from B, C, and D. B's transmission will start after it receives A's beacon signal; C's transmission will start after it receives both A and B's beacon signals; And D's transmission will start after it receives A, B, and C's beacon signals. This procedure will be repeated once every $T$ seconds.

\section{Step 1: Range Difference Computation.}

Let $\mathrm{A}$ be the master anchor node, which initiates a beacon signal every $T$ seconds. Each beacon interval begins when A transmits a beacon signal. Consider any beacon interval $i$, at times $t_{1}^{i}, t_{b}^{i}$, $t_{c}^{i}$, and $t_{d}^{i}$, sensor $S$, anchor nodes $\mathrm{B}, \mathrm{C}$, and $\mathrm{D}$ receive A's beacon signal, respectively. At time $t_{b}^{i \prime}$, which is $\geq t_{b}^{i}$, B replies to A with a beacon signal conveying information $t_{b}^{i \prime}-t_{b}^{i}=\Delta t_{b}^{i}$. This signal reaches $S$ at time $t_{2}^{i}$. After receiving beacon signals from both A and $\mathrm{B}$, at time $t_{c}^{i \prime}, \mathrm{C}$ replies to $\mathrm{A}$ with a beacon signal conveying information $t_{c}^{i \prime}-t_{c}^{i}=\Delta t_{c}^{i}$. This signal reaches $S$ at time $t_{3}^{i}$. After receiving beacon signals from $\mathrm{A}, \mathrm{B}$, and $\mathrm{C}$, at time $t_{d}^{i \prime}$, D replies to $\mathrm{A}$ with a beacon signal conveying information $t_{d}^{i j}-t_{d}^{i}=\Delta t_{d}^{i}$. This signal reaches $S$ at time $t_{4}^{i}$. Based on triangle inequality, $t_{1}^{i}<t_{2}^{i}<t_{3}^{i}<t_{4}^{i}$. Let 
$\Delta t_{1}^{i}=t_{2}^{i}-t_{1}^{i}, \Delta t_{2}^{i}=t_{3}^{i}-t_{1}^{i}$, and $\Delta t_{3}^{i}=t_{4}^{i}-t_{1}^{i}$, we obtain

$$
\begin{aligned}
& d_{a b}+d_{s b}-d_{s a}+v \cdot \Delta t_{b}^{i}=v \cdot \Delta t_{1}^{i}, \\
& d_{a c}+d_{s c}-d_{s a}+v \cdot \Delta t_{c}^{i}=v \cdot \Delta t_{2}^{i}, \\
& d_{a d}+d_{s d}-d_{s a}+v \cdot \Delta t_{d}^{i}=v \cdot \Delta t_{3}^{i},
\end{aligned}
$$

which gives

$$
\begin{aligned}
d_{s b} & =d_{s a}+v \cdot \Delta t_{1}^{i}-d_{a b}-v \cdot \Delta t_{b}^{i}=d_{s a}+k_{1}^{i}, \\
d_{s c} & =d_{s a}+v \cdot \Delta t_{2}^{i}-d_{a c}-v \cdot \Delta t_{c}^{i}=d_{s a}+k_{2}^{i}, \\
d_{s d} & =d_{s a}+v \cdot \Delta t_{3}^{i}-d_{a d}-v \cdot \Delta t_{d}^{i}=d_{s a}+k_{3}^{i},
\end{aligned}
$$

where $d_{s a}, d_{s b}, d_{s c}$, and $d_{s d}$ are positive real numbers, $v$ is the speed of the ultrasound, and

$$
\begin{aligned}
k_{1}^{i} & =v \cdot \Delta t_{1}^{i}-v \cdot \Delta t_{b}^{i}-d_{a b} \\
k_{2}^{i} & =v \cdot \Delta t_{2}^{i}-v \cdot \Delta t_{c}^{i}-d_{a c} \\
k_{3}^{i} & =v \cdot \Delta t_{3}^{i}-v \cdot \Delta t_{d}^{i}-d_{a d} .
\end{aligned}
$$

Averaging $k_{1}^{i}, k_{2}^{i}$, and $k_{3}^{i}$ over $I$ intervals gives

$$
\begin{aligned}
& k_{1}=\frac{v}{I}\left[\sum_{i=1}^{I}\left(\Delta t_{1}^{i}-\Delta t_{b}^{i}\right)\right]-d_{a b}, \\
& k_{2}=\frac{v}{I}\left[\sum_{i=1}^{I}\left(\Delta t_{2}^{i}-\Delta t_{c}^{i}\right)\right]-d_{a c}, \\
& k_{3}=\frac{v}{I}\left[\sum_{i=1}^{I}\left(\Delta t_{3}^{i}-\Delta t_{d}^{i}\right)\right]-d_{a d} .
\end{aligned}
$$

We are going to apply trilateration with $k_{1}, k_{2}$, and $k_{3}$ to compute coordinates $(x, y, z)$ for sensor $S$ in the next step.

Remarks: (i) All arrival times, including $t_{j}^{i}$, where $j=1,2,3,4$, and $t_{j}^{i \prime}$, where $j \in\{b, c, d\}$, are based on the local timers of the anchor nodes and the sensor $S$. No time synchronization is required. (ii) We require A to periodically initiate the beacon signal transmission in order to decrease the measurement error and to facilitate navigation.

Step 2: Location Computation.

From Eqs. (4), (5), (6), (10), (11), and (12), we have

$$
\begin{aligned}
d_{s b} & =d_{s a}+k_{1}, \\
d_{s c} & =d_{s a}+k_{2}, \\
d_{s d} & =d_{s a}+k_{3} .
\end{aligned}
$$

Based on trilateration, we obtain four equations with four unknowns $x, y, z$ and $d_{s a}$, where $d_{s a}>0$.

$$
\begin{aligned}
\left(x-x_{a}\right)^{2}+\left(y-y_{a}\right)^{2}+\left(z-z_{a}\right)^{2} & =d_{s a}^{2}, \\
\left(x-x_{b}\right)^{2}+\left(y-y_{b}\right)^{2}+\left(z-z_{b}\right)^{2} & =\left(d_{s a}+k_{1}\right)^{2}, \\
\left(x-x_{c}\right)^{2}+\left(y-y_{c}\right)^{2}+\left(z-z_{c}\right)^{2} & =\left(d_{s a}+k_{2}\right)^{2}, \\
\left(x-x_{d}\right)^{2}+\left(y-y_{d}\right)^{2}+\left(z-z_{d}\right)^{2} & =\left(d_{s a}+k_{3}\right)^{2} .
\end{aligned}
$$

Without loss of generality, we assume that the four anchor nodes are located at $(0,0,0),\left(x_{b}, 0,0\right),\left(x_{c}, y_{c}, 0\right)$, and $\left(x_{d}, y_{d}, z_{d}\right)$, respectively, where $x_{b}>0, y_{c}>0$, and $z_{d}>0$. Note that we can always transform real positions to this coordinate system through rotation and translation. 
From Eqs. (16), (17), (18), and (19), we have

$$
\begin{gathered}
x^{2}+y^{2}+z^{2}=d_{s a}^{2}, \\
\left(x-x_{b}\right)^{2}+y^{2}+z^{2}=\left(d_{s a}+k_{1}\right)^{2}, \\
\left(x-x_{c}\right)^{2}+\left(y-y_{c}\right)^{2}+z^{2}=\left(d_{s a}+k_{2}\right)^{2}, \\
\left(x-x_{d}\right)^{2}+\left(y-y_{d}\right)^{2}+\left(z-z_{d}\right)^{2}=\left(d_{s a}+k_{3}\right)^{2},
\end{gathered}
$$

Solving these equations we obtain

$$
\begin{aligned}
d_{s a}^{(1)} & =\frac{-\beta-\sqrt{\beta^{2}-4 \alpha \gamma}}{2 \alpha} \\
d_{s a}^{(2)} & =\frac{-\beta+\sqrt{\beta^{2}-4 \alpha \gamma}}{2 \alpha} \\
x & =A_{x} d_{s a}+B_{y} \\
y & =A_{y} d_{s a}+B_{y} \\
z & =A_{z} d_{s a}+B_{z}
\end{aligned}
$$

where

$$
\begin{aligned}
\alpha & =A_{x}^{2}+A_{y}^{2}+A_{z}^{2}-1 \\
\beta & =2\left(A_{x} B_{x}+A_{y} B_{y}+A_{z} B_{z}\right) \\
\gamma & =B_{x}^{2}+B_{y}^{2}+B_{z}^{2} \\
A_{x} & =-\frac{k_{1}}{x_{b}} \\
B_{x} & =\frac{x_{b}^{2}-k_{1}^{2}}{2 x_{b}} \\
A_{y} & =\frac{k_{1} x_{c}}{x_{b} y_{c}}-\frac{k_{2}}{y_{c}} \\
B_{y} & =\frac{x_{c}^{2}+y_{c}^{2}-x_{b} x_{c}+\frac{x_{c} k_{1}^{2}}{x_{b}}-k_{2}^{2}}{2 y_{c}} \\
A_{z} & =\frac{k_{1} x_{d}}{x_{b} z_{d}}-\frac{k_{3}}{z_{d}}-\frac{y_{d}\left(\frac{k_{1} x_{c}}{x_{b}}-k_{2}\right)}{y_{c} z_{d}} \\
B_{z} & =\frac{x_{d}^{2}+y_{d}^{2}+z_{d}^{2}-x_{b} x_{d}+\frac{x_{d} k_{1}^{2}}{x_{b}}-k_{3}^{2}-\frac{y_{d} x_{c}^{2}}{y_{c}}}{2 z_{d}} \\
& +\frac{-y_{c} y_{d}+\frac{x_{b} x_{c} y_{d}}{y_{c}}-\frac{k_{1}^{2} x_{c} y_{d}}{x_{b} y_{c}}+\frac{k_{2}^{2} y_{d}}{y_{c}}}{2 z_{d}}
\end{aligned}
$$

We have conducted extensive simulation to study the feasible space where $d_{s a}>0$ is unique. It is interesting to observe that when $S$ is not close to any anchor node, and when it is not behind any anchor node, Eq. (24) provides a unique feasible solution. In addition, the correct position can be computed via Eq. (24) if a sensor resides in the enclosed space by the four anchor nodes, even when it is close to an anchor node. Figs. 2-4 report the three transections $(z=0,5,10)$ of the feasible space (the gray area) when the four anchor nodes $\mathrm{A}, \mathrm{B}, \mathrm{C}$, and $\mathrm{D}$ reside in $(0,0,0),(10,0,0),(0,10,0)$, and $(0,0,10)$, respectively.

\section{Channel Modeling and Theoretical Performance Analysis}

In this section, we study the position error of UPS resulted from the acoustic fading channel. In the following, we first propose a modified UWB S-V model to characterize the underwater acoustic fading channel, then we apply this model to study the position error of UPS. Major sources of position errors are also identified. Note that to our best knowledge, there is no standard channel model for underwater so far and based on our study [20], this modified UWB S-V model is a reasonable one. A detailed studv on this model is available at [20]. 


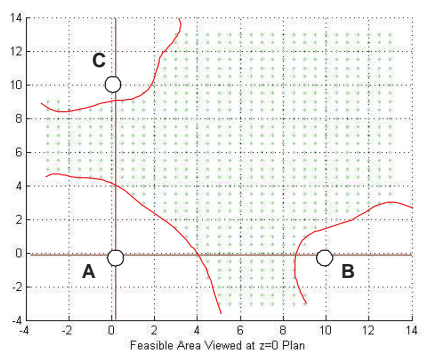

Figure 2: The transection of the feasible space where $z=0$

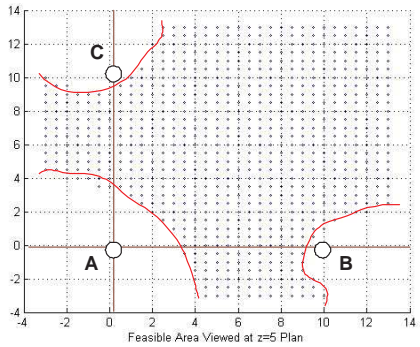

Figure 3: The transection of the feasible space where $z=5$

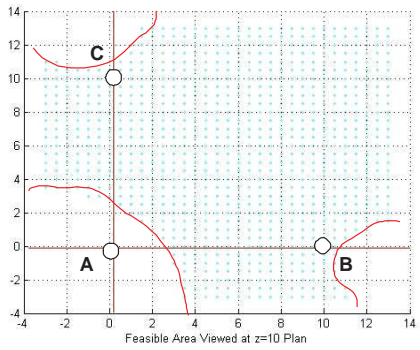

Figure 4: The transection of the feasible space where $z=10$

\subsection{Channel Modeling for Underwater Sensor Networks}

The underwater acoustic channel exhibits phenomena such as signal fading and phase and amplitude fluctuation due to the interactions with the boundaries and the scattering from inhomogeneities within the ocean medium. The speed of sound underwater is approximately $1500 \mathrm{~m} / \mathrm{s}$, which leads to large propagation delays and motion-induced Doppler effects. Phase and amplitude fluctuations may induce high bit-error probability comparing to most radio channels. Multipath interference is another important phenomena in UWA networks, causing frequency selective fading in underwater channels.

There have been a lot of effort to model the underwater acoustic fading channels and estimate their performance. Early research [12][25] assumes Rayleigh fading in nature but later it is observed in [16] that Rayleigh fading exhibits only in limited cases. Geng and Zielinski [16] propose that in an underwater acoustic channel, there can be several distinct paths (eigenpaths) over which a signal can propagate from transmitter to receiver (eigenpath signals). Each eigenpath signal contains a dominant, stable component and many smaller, randomly scattered components (subeigenpath or engenray components). The envelope of the eigenpath signal can therefore be described using a Rice fading model. Such an eigenpath or eigenray concept was first introduced in [18]. Eigenray arrival angles as well as the amplitude and phase fluctuations are all modelled statistically and are assumed independent from each other. The number of eigenrays reaching the receiver is a Poisson distribution with a mean number calculated from the Ray Theory.

Enlightened by the prior research on underwater acoustic channel modeling, we propose in this paper a modified UWB Saleh-Valenzuela (S-V) channel model for underwater acoustic networks, which can be validated in three considerations. First. UWB comes from the UWB radar world and refers to the electromagnetic waveforms that are 
characterized by an instantaneous fractional energy bandwidth greater than about $0.20-0.25$. In an underwater acoustic channel, the communication frequency range is inferior to $10 \mathrm{kHz}$. In short range transmission, the carrier frequency is $550 \mathrm{~Hz}$ in shallow water and $2 \mathrm{kHz}$ in deep water. The carrier frequency for long range transmission is $1500 \mathrm{~Hz}$. In all cases, the fractional bandwidth $\left(f_{H}-f_{L}\right) /\left(\left(f_{H}+f_{L}\right) / 2\right)$ is greater than $0.20-0.25$. Therefore, the acoustic signal transmitted underwater can be classified as a UWB signal. Second, Multipath channel can be modelled as a S-V model in UWB communications. The S-V model is based on the observation that usually multipath contributions generated by the same pulse arrive at the receiver grouped into clusters. Such a channel behavior is analogous to the above eigenpath/sub-eigenpath concept in underwater networks. Third, Similar to [18], two Poisson models are employed in the modeling of the path arrivals in UWB communications. The first Poisson model is for the first path of each path cluster and the second Poisson model is for the paths or rays within each cluster.

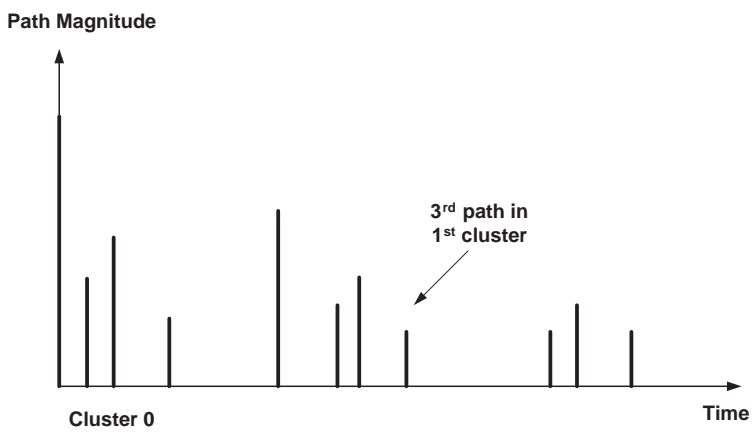

Figure 5: An illustration of the channel impulse response

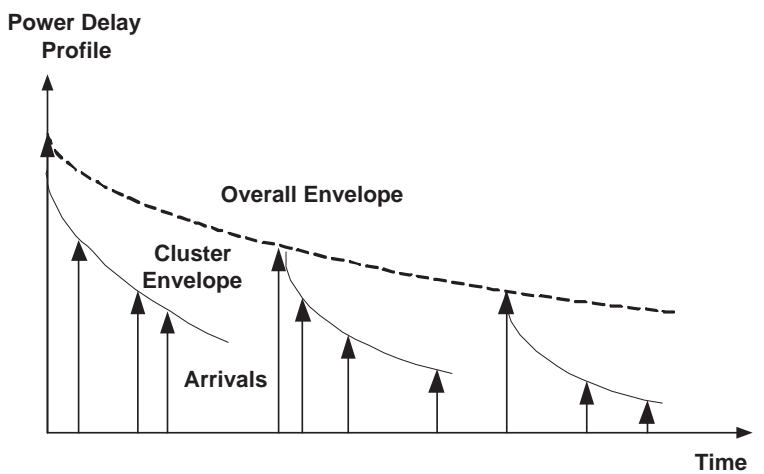

Figure 6: An illustration of the double exponential decay of the mean cluster power and the ray power within clusters

Applying the S-V model into underwater acoustic channels, the arrival of clusters is modelled as a Poisson arrival process with a rate $\Lambda$, while within each cluster, subsequent multipath contributions or rays also arrive according to a Poisson process with a rate $\lambda$ (see Fig. 5). We define:

- $T_{l}=$ the arrival time of the first path of the $l$-th cluster;

- $\tau_{k, l}=$ the delay of the $k$-th path within the $l$-th cluster relative to the first path arrival time $T_{l}$;

- $\Lambda=$ the cluster arrival rate;

- $\lambda=$ the ray arrival rate, i.e., the arrival rate of the paths within each cluster.

By definition, we have $\tau_{0 l}=T_{l}$. The distributions of the cluster arrival time and the ray arrival time are given by

$$
\begin{array}{r}
p\left(T_{l} \mid T_{l-1}\right)=\Lambda \exp \left(-\Lambda\left(T_{l}-T_{l-1}\right), l>0\right. \\
p\left(\tau_{k, l} \mid \tau_{(k-1), l}\right)=\lambda \exp \left(-\lambda\left(\tau_{k, l}-\tau_{(k-1), l}\right)\right), k>0
\end{array}
$$

In the UWB S-V model, the magnitude of the $k$-th path within the $l$-th cluster is denoted by $\beta_{k l}$. It follows a Ravleigh distribution. However in underwater acoustic networks the channel within the communication freauencv 
range does not behave like a Rayleigh channel. Based on the discussion in $[16,18]$, it is rather appropriate to model the multipath channel gain as a Rician distribution. Then in the underwater S-V model, the gain of the $k$-th path within the $l$-th cluster is a complex random value with a modulus $\beta_{k l}$ and a phase $\theta_{k l}$. We assume that the $\beta_{k l}$ values in a underwater acoustic channel are statistically independent and are Rician distributed positive random variables, while the $\theta_{k l}$ values are assumed to be statistically independent uniform random variables over $[0,2 \pi)$. We have

$$
\overline{\beta_{k l}^{2}}=\overline{\beta_{00}^{2}} \exp \left(-T_{l} / \Gamma\right) \exp \left(-\tau_{k l} / \gamma\right)
$$

where the term $\beta_{00}$ represents the average energy of the first path of the first cluster, while $\Gamma$ and $\gamma$ are the power decay coefficients for clusters and multipath, respectively. According to (39), the average Power Decay Profile (PDP) is characterized by an exponential decay of the amplitude of the clusters, and a different exponential decay for the amplitude of the received pulses within each cluster, as shown in Fig. 6.

\subsection{Theoretical Error Analysis}

In this subsection, we study the position error of $S$ resulted from the acoustic fading channel, which has been modelled as a modified UWB S-V model in Subsection 4.1.

The trilateration equations (16)-(19) compute the coordinates $(x, y, z)$ for the sensor $S$ based on the measured values $k_{1}, k_{2}$, and $k_{3}$, which are determined by the time-related measurements at the sensor $\left(\Delta t_{1}^{i}, \Delta t_{2}^{i}\right.$ and $\left.\Delta t_{3}^{i}\right)$ and anchor nodes $\mathrm{B}\left(\Delta t_{b}^{i}\right), \mathrm{C}\left(\Delta t_{c}^{i}\right)$, and $\mathrm{D}\left(\Delta t_{d}^{i}\right)$ over beacon interval $i$ (see Eqs. (10)-(12)). Therefore the errors of $x$, $y$ and $z$ result from the measuring errors of $\Delta t_{1}^{i}, \Delta t_{2}^{i}, \Delta t_{3}^{i}, \Delta t_{b}^{i}, \Delta t_{c}^{i}$, and $\Delta t_{d}^{i}$. Since the error of $\Delta t_{b}^{i}\left(\Delta t_{c}^{i}, \Delta t_{d}^{i}\right)$ plays the same role as that of $\Delta t_{1}^{i}\left(\Delta t_{2}^{i}, \Delta t_{3}^{i}\right)$ in the computation of $k_{1}\left(k_{2}, k_{3}\right)$, and the anchor node B (C, D) can have more sophisticated hardware to precisely estimate $\Delta t_{b}^{i}\left(\Delta t_{c}^{i}, \Delta t_{d}^{i}\right)$, we consider the errors of $\Delta t_{1}^{i}, \Delta t_{2}^{i}$ and $\Delta t_{3}^{i}$ only, which are computed from $t_{1}^{i}, t_{2}^{i}, t_{3}^{i}$, and $t_{4}^{i}$, the arrival times of the beacon signals transmitted from anchor nodes $\mathrm{A}, \mathrm{B}, \mathrm{C}$, and $\mathrm{D}$ at beacon internal $i$, respectively.

Assume the underwater sensor always listens to the first ray of the transmitted signal and records the arrival times, which in our case are $t_{1}^{i}, t_{2}^{i}, t_{3}^{i}$ and $t_{4}^{i}$. Due to the underwater multi-path effect as illustrated in Fig. 6, these arrival times contain an error with an exponential distribution. Let $\delta_{t 1}^{i}, \delta_{t 2}^{i}$, and $\delta_{t 3}^{i}$ be the measuring errors of $\Delta t_{1}^{i}$, $\Delta t_{2}^{i}$, and $\Delta t_{3}^{i}$, respectively. It is reasonable to assume that $\delta_{t 1}^{i}, \delta_{t 2}^{i}$, and $\delta_{t 3}^{i}$ are independent to each other. Given $t_{1}^{i}$, the conditional probability density functions $(p d f)$ of $\delta_{t 1}{ }_{1}^{i}, \delta_{t 2}$, and $\delta_{t 3}^{i}$ are exponential with parameters $\lambda_{1}, \lambda_{2}$ and $\lambda_{3}$, respectively:

$$
\begin{aligned}
& P_{e}\left(\delta_{t 1}^{i} \mid t_{1}^{i}\right)=\lambda_{1} \exp \left(-\lambda_{1} \delta_{t}^{i}\right), \\
& P_{e}\left(\delta_{t 2}^{i} \mid t_{1}^{i}\right)=\lambda_{2} \exp \left(-\lambda_{2} \delta_{t 2}^{i}\right), \\
& P_{e}\left(\delta_{t 3}^{i} \mid t_{1}^{i}\right)=\lambda_{3} \exp \left(-\lambda_{3} \delta_{t 3}^{i}\right) .
\end{aligned}
$$

To simplify the elaboration, we consider the case when anchor nodes A, B, C, and D are located at $(0,0,0)$, $(R, 0,0),(0, R, 0)$, and $(0,0, R)$, respectively. To further simplify the analysis, we consider the case when $S$ resides in a small area (with a diameter $\ll R$ ) whose center is equidistant to all anchor nodes. The general case can be analyzed similarly.

From Eqs. (10)-(12), $k_{1}, k_{2}$, and $k_{3}$ are the averaged results over $I$ beacon intervals, and based on the Central Limit Theorem, $k_{1}, k_{2}$ and $k_{3}$ are approximately normally distributed when $I$ is large. Therefore we may assume $k_{1}, k_{2}$, and $k_{3}$ are distributed according to $\mathcal{N}\left(\mu_{1}, \sigma_{1}^{2}\right), \mathcal{N}\left(\mu_{2}, \sigma_{2}^{2}\right)$, and $\mathcal{N}\left(\mu_{3}, \sigma_{3}^{2}\right)$, respectively. Deducing from Eqs. (10)-(12), we have

$$
\begin{array}{ll}
k_{1}: \mu_{1}=v\left(\frac{1}{\lambda_{1}}+\nu_{1}\right)-R, & \sigma_{1}^{2}=\frac{v^{2}}{I \lambda_{1}^{2}}, \\
k_{2}: \mu_{2}=v\left(\frac{1}{\lambda_{2}}+\nu_{2}\right)-R, & \sigma_{2}^{2}=\frac{v^{2}}{I \lambda_{2}^{2}}, \\
k_{3}: \mu_{3}=v\left(\frac{1}{\lambda_{3}}+\nu_{3}\right)-R, & \sigma_{3}^{2}=\frac{v^{3}}{I \lambda_{3}^{2}} .
\end{array}
$$

where $\nu_{1}, \nu_{2}$ and $\nu_{3}$ are the mean of the accurate values for $\Delta t_{1}^{i}, \Delta t_{2}^{i}$ and $\Delta t_{3}^{i}$, respectively. 
Based on the assumptions, we have $\mu_{1} / R \approx 0, \mu_{2} / R \approx 0$ and $\mu_{3} / R \approx 0$. Plugging $x_{b}=y_{c}=z_{d}=R$ and other zero coordinates into Eq. (24) and simplify its solution by approximating $k_{1}^{2} / R^{2}, k_{2}^{2} / R^{2}$ and $k_{3}^{2} / R^{2}$ with 0 , we end up with

$$
d_{s a} \approx \frac{\sqrt{3 R^{2}+\left(k_{1}+k_{2}+k_{3}\right)^{2}}-\left(k_{1}+k_{2}+k_{3}\right)}{2} .
$$

Substituting the above into Eq. (26) yields

$$
x \approx \frac{R}{2}-\frac{k_{1}^{2}}{2 R}-\frac{k_{1}}{R} \frac{\sqrt{3 R^{2}+\left(k_{1}+k_{2}+k_{3}\right)^{2}}-\left(k_{1}+k_{2}+k_{3}\right)}{2} .
$$

Now replacing $\frac{k_{1} k_{2}}{R^{2}}=\frac{k_{1}}{R} \frac{k_{2}}{R}, \frac{k_{1} k_{3}}{R^{2}}=\frac{k_{1}}{R} \frac{k_{3}}{R}$ and $\frac{k_{2} k_{3}}{R^{2}}=\frac{k_{2}}{R} \frac{k_{3}}{R}$ by 0 , we obtain

$$
x \approx \frac{R}{2}+\frac{k_{1}}{2 R}\left(k_{2}+k_{3}\right)-k_{1} \sqrt{\frac{3}{2}}=\frac{R}{2}+k_{1} k_{2,3}^{*},
$$

where $k_{2,3}^{*}=\left(k_{2}+k_{3}\right) / 2 R-\sqrt{3 / 2}$. Similarly, from Eq. (27) and (28) we have

$$
\begin{aligned}
y & \approx \frac{R}{2}+\frac{k_{2}}{2 R}\left(k_{1}+k_{3}\right)-k_{2} \sqrt{\frac{3}{2}}=\frac{R}{2}+k_{2} k_{1,3}^{*}, \\
z & \approx \frac{R}{2}+\frac{k_{3}}{2 R}\left(k_{1}+k_{2}\right)-k_{3} \sqrt{\frac{3}{2}}=\frac{R}{2}+k_{3} k_{1,2}^{*} .
\end{aligned}
$$

where $k_{1,3}^{*}=\left(k_{1}+k_{3}\right) / 2 R-\sqrt{3 / 2}$, and $k_{1,2}^{*}=\left(k_{1}+k_{2}\right) / 2 R-\sqrt{3 / 2}$.

Since $(x, y, z)$ is used to estimate the location of $S$, the error in the estimation must be addressed. There are several ways to do this. The following is a common practice, where the variance of each variable is computed and the size of the variance or standard deviation is used as a measure of the estimation error.

As $k_{1}$ has a Gaussian distribution with mean $\mu_{1}$ and variance $\sigma_{1}^{2}, k_{2}$ has a Gaussian distribution with mean $\mu_{2}$ and variance $\sigma_{2}^{2}$, and $k_{3}$ has a Gaussian distribution with mean $\mu_{3}$ and variance $\sigma_{3}^{2}$, the linear combination $k_{1,3}^{*}$ has a Gaussian distribution with mean $\left(\mu_{1}+\mu_{3}\right) / 2 R-\sqrt{3 / 2}$ and variance $\left(\sigma_{1}^{2}+\sigma_{3}^{2}\right) / 4 R^{2}, k_{1,2}^{*}$ has a Gaussian distribution with mean $\left(\mu_{1}+\mu_{2}\right) / 2 R-\sqrt{3 / 2}$ and variance $\left(\sigma_{1}^{2}+\sigma_{2}^{2}\right) / 4 R^{2}$, and $k_{2,3}^{*}$ has a Gaussian distribution with mean $\left(\mu_{2}+\mu_{3}\right) / 2 R-\sqrt{3 / 2}$ and variance $\left(\sigma_{2}^{2}+\sigma_{3}^{2}\right) / 4 R^{2}$. Denote by $E(X)$ and $V(X)$ the mean and variance of a random variable $X$. We have, from Eq. (44),

$$
\begin{aligned}
V(x) & \approx V\left(k_{1} k_{2,3}^{*}\right) \\
& =E\left(k_{1} k_{2,3}^{*}\right)^{2}-\left[E\left(k_{1} k_{2,3}^{*}\right)\right]^{2} \\
& =E\left(k_{1}^{2}\left(k_{2,3}^{*}\right)^{2}\right)-\left[E\left(k_{1} k_{2,3}^{*}\right)\right]^{2} .
\end{aligned}
$$

By the independence between $k_{1}, k_{2}$ and $k_{3}$, we have

$$
\begin{aligned}
E\left(k_{1} k_{2,3}^{*}\right)= & E\left(k_{1}\right) E\left(k_{2,3}^{*}\right) \\
E\left(k_{1}^{2}\left(k_{2,3}^{*}\right)^{2}\right)= & E\left(k_{1}^{2}\right) E\left(k_{2,3} *^{2}\right) \\
= & {\left[V\left(k_{1}\right)+\left(E\left(k_{1}\right)\right)^{2}\right] } \\
& {\left[V\left(k_{2,3}^{*}\right)+\left(E\left(k_{2,3}^{*}\right)\right)^{2}\right] . }
\end{aligned}
$$


Therefore substitution gives

$$
\begin{aligned}
V(x) \approx & V\left(k_{1}\right)\left[E\left(k_{2,3}^{*}\right)\right]^{2}+V\left(k_{2,3}^{*}\right)\left[E\left(k_{1}\right)\right]^{2} \\
& +V\left(k_{1}\right) V\left(k_{2,3}^{*}\right) \\
= & \sigma_{1}^{2}\left(\frac{\mu_{2}+\mu_{3}}{2 R}-\sqrt{\frac{3}{2}}\right)^{2}+\frac{\sigma_{2}^{2}+\sigma_{3}^{2}}{4 R^{2}} \mu_{1}^{2} \\
& +\sigma_{1}^{2} \frac{\sigma_{2}^{2}+\sigma_{3}^{2}}{4 R^{2}} \\
= & \frac{\sigma_{1}^{2}\left(\mu_{2}+\mu_{3}\right)^{2}+\left(\sigma_{2}^{2}+\sigma_{3}^{2}\right) \mu_{1}^{2}+\sigma_{1}^{2}\left(\sigma_{2}^{2}+\sigma_{3}^{2}\right)}{4 R^{2}} \\
& +\sigma_{1}^{2}\left(\frac{3}{2}-\frac{\mu_{2}+\mu_{3}}{R} \sqrt{\frac{3}{2}}\right) .
\end{aligned}
$$

Since $\mu_{1} / R \approx 0, \mu_{2} / R \approx 0$, plugging in Eq.(42), the above reduces to

$$
\begin{aligned}
V(x) & \approx \frac{\sigma_{1}^{2}}{2}\left(3+\frac{\sigma_{2}^{2}+\sigma_{3}^{2}}{2 R^{2}}\right) \\
& =\frac{v^{2}}{2 I \lambda_{1}^{2}}\left[3+\frac{v^{2}}{2 I R\left(\lambda_{2}^{2}+\lambda_{3}^{2}\right)}\right] .
\end{aligned}
$$

Similarly, we have

$$
\begin{aligned}
V(y) \approx & V\left(k_{1,3}^{*}\right)\left[E\left(k_{2}\right)\right]^{2}+V\left(k_{2}\right)\left[E\left(k_{1,3}^{*}\right)\right]^{2} \\
& +V\left(k_{1,3}^{*}\right) V\left(k_{2}\right) \\
\approx & \frac{\sigma_{2}^{2}}{2}\left(3+\frac{\sigma_{1}^{2}+\sigma_{3}^{2}}{2 R^{2}}\right) \\
= & \frac{v^{2}}{2 I \lambda_{2}^{2}}\left[3+\frac{v^{2}}{2 I R\left(\lambda_{1}^{2}+\lambda_{3}^{2}\right)}\right] .
\end{aligned}
$$

and

$$
\begin{aligned}
V(z) \approx & V\left(k_{1,2}^{*}\right)\left[E\left(k_{3}\right)\right]^{2}+V\left(k_{3}\right)\left[E\left(k_{1,2}^{*}\right)\right]^{2} \\
& +V\left(k_{1,2}^{*}\right) V\left(k_{3}\right) \\
\approx & \frac{\sigma_{3}^{2}}{2}\left(3+\frac{\sigma_{1}^{2}+\sigma_{2}^{2}}{2 R^{2}}\right) \\
= & \frac{v^{2}}{2 I \lambda_{3}^{2}}\left[3+\frac{v^{2}}{2 I R\left(\lambda_{1}^{2}+\lambda_{2}^{2}\right)}\right] .
\end{aligned}
$$

From the above analysis, we have the following observations. First, the variances of $x, y$ and $z$ depend on the ray arrival rates $\lambda_{1}, \lambda_{2}$ and $\lambda_{3}$. Second, $\lambda_{1}$ contributes more to the variance of $x$ than $\lambda_{2}$ and $\lambda_{3}, \lambda_{2}$ contributes more to the variance of $y$ than $\lambda_{1}$ and $\lambda_{3}$, and $\lambda_{3}$ contributes more to the variance of $z$ than $\lambda_{1}$ and $\lambda_{2}$. Third, when $R$ is large, $V(x) \approx 3 \sigma_{1}^{2} / 2=3 v^{2} / 2 I \lambda_{1}^{2}, V(y) \approx 3 \sigma_{2}^{2} / 2=3 v^{2} / 2 I \lambda_{2}^{2}$, and $V(z) \approx 3 \sigma_{3}^{2} / 2=3 v^{2} / 2 I \lambda_{3}^{2}$, showing that the variance of $x$ is dependent on that of $k_{1}$, the variance of $y$ is dependent on that of $k_{2}$, and the variance of $z$ is dependent on that of $k_{3}$. Fourth, if $\lambda_{1}=\lambda_{2}=\lambda_{3}$, the variances of $x, y$ and $z$ can be treated the same in practice.

Note that the above analysis well-explain our simulation results, which indicate position errors strongly depend on the arrival rates of double exponential distributions.

\subsection{Sources of Errors}

There are three major sources of errors for time-based location detection schemes in UWA-SNs: the receiver system delay, the underwater multipath fading, and the variable acoustic speed underwater. The receiver system delay is the time duration from which the sional hits the receiver antenna until the sional is decoded accuratelv by the 
receiver. This time delay is determined by the receiver electronics. Usually it is constant or varies in very small scale when the receiver and the channel are free from interference. This system delay can be predetermined and be used to calibrate the measurements. For example, anchor nodes B, C, and D can always eliminate the system delay from $\Delta t_{b}^{i}, \Delta t_{c}^{i}$ and $\Delta t_{d}^{i}$ before these values are conveyed to the sensors in their reply messages to A's beacon signal. Meanwhile, as $\Delta t_{1}^{i}, \Delta t_{2}^{i}$ and $\Delta t_{3}^{2}$ are measured by one sensor, the effect of receiver system delay may cancel out. Thus in our model, if anchor nodes B, C, and D can provide precise a priori information on receiver system time delays, the effect of these delays will be negligible.

The underwater multipath fading channel will greatly influence the location accuracy of any location detection system. Major factors influencing terrestrial multipath fading [5] include multipath propagation, speed of the receiver, speed of the surrounding objects, and the transmission signal bandwidth. In the underwater environment, other important factors include water temperature and clarity, motion behavior of receiver and underwater objects and transmission range. In our time-based location scheme, we assume that the motion of the underwater vehicles is relatively small such that the motion-induced Doppler effect can be ignored.

There are two important characteristics of multipath signals. First, the multiple non-direct path signals will always arrive at the receiver antennae latter than the direct path signal, as they must travel a longer distance. Second, in LOS transmission model, non-direct multipath signals will normally be weaker than the direct path signal, as some signal power will be lost from scattering. If NLOS exists, the non-direct multipath signal may be stronger, as the direct path is hindered in some way. Based on these characteristics, scientists can always design more sensitive receivers to lock and track the direct path signal. For example, multipath signals using a pseudo-random code arriving at the receiver later than the direct path signal will have negligible effects on a high-resolution DS-BPSK receiver [10]. Our location detection scheme mitigates the effect of multipath fading by measuring TDoA over multiple beacon intervals and modeling the multipath arrival times as the double exponential distribution. TDoA measurements have been very effective in fading channels, as many detrimental effects caused by multipath fading and processing delay can be cancelled [11].

Another source of position error is the variable speed of sound, which significantly affects the precision of all localization systems that assume a constant acoustic speed. The velocity of underwater acoustics depends on temperature, salinity, and depth [3]. As a future research, we will investigate the influence of the variable speed of sound on our positioning scheme.

\section{Simulation}

In this section, we are going to study the performance of UPS in UWA-SN. We take the same assumptions as those in Subsection 4.2, that for a given $t_{1}^{i}$, the measuring errors of $\Delta t_{i}^{i}, \Delta t_{2}^{i}$ and $\Delta t_{3}^{i}$ are independent exponential distributions with arrival rates $\lambda_{1}, \lambda_{2}$ and $\lambda_{3}$, respectively, and that the underwater vehicle/sensor measures the arrival time of the first ray of the first cluster only in a multipath fading channel. We further assume that the measuring errors of $\Delta t_{b}^{i}, \Delta t_{c}^{i}$ and $\Delta t_{d}^{i}$ are negligible, as justified in Subsection 4.3. Therefore $\lambda_{1}, \lambda_{2}$ and $\lambda_{3}$ can be assumed to be equal, and this value is denoted by $\lambda$. We will investigate the influence of $\lambda$ upon position error. Another factor we will investigate is the number of beacon intervals $I$ used to compute $k_{1}, k_{2}$, and $k_{3}$. Since we also consider the localization of mobile underwater vehicles, we choose to average over a small number of beacon intervals.

We use Matlab to code UPS. This tool provides procedures to generate normally distributed and uniformly distributed random numbers. Note that we do not use the sqrt function in Matlab. Instead, we use Newton's method [2]. We have found that 4 iterations generally yield good results. In addition, Eq. (24) will be adopted for position estimation since in our simulation, sensors will be placed within the space enclosed by four anchor nodes.

First we study the distribution of position errors over a 3D monitored space. In this simulation scenario, the four anchor nodes are located at $(0,0,0),(20,0,0),(0,20,0)$, and $(0,0,20)$ respectively. Sensors are placed at grid points $(i+0.5, j+0.5, k+0.5)$, where $i, j, k=0,1, \cdots, 19$. The errors of $\Delta t_{1}^{i}, \Delta t_{2}^{i}$ and $\Delta t_{3}^{i}$ are exponentially distributed with parameters $\lambda_{1}=\lambda_{2}=\lambda_{3}=\lambda$. We average the sensor location estimation over 1000 trials. For each trial, $I=16$. In addition, we simulate different $\lambda$ settings and obtain similar results. Nevertheless, we report the cases of $\lambda=0.1$ and $\lambda=2.5$ only in this paper. Fig. 7 and Fig. 8 illustrate the $(x-y)$ plane position errors vs. the real positions of the sensors for $z=5$. Results from $(x-z)$ and $(y-z)$ planes are close to those reported for the $(x-y)$ plane.

We observe that a lower arrival rate gives a better estimation since a higher arrival rate may bring motion-induced 
Doppler shift in the channel and cause jittering in the measurement. We also observe that as the distances from a sensor to all four anchor nodes become larger, the position errors will become larger correspondingly; when the sensor is closer to any of the four anchor nodes, the errors become larger. Notice that in Fig. 7 the sensor at location $(9.5,9.5,5.0)$, which is close to the intersection of the three angle bisectors of $\triangle A B C$, has the smallest position error and the sensors at its neighboring area also demonstrate quite low position errors. Interestingly, Refs. [9, 15, 22] provide similar results in their simulation study. Intuitively this is because the geometry of the intersection of the range circles is poor when the sensors are far away from any anchor node or when the sensors are close to any anchor node. From this analysis, we conclude that the position error is related to the placement of anchor nodes. Careful studies will be conducted in the future as the results can be applied to guide the deployment of anchor nodes for better performance. For these reasons, in the following simulation, we intentionally enforce an allowable shortest distance (1.0 unit) from any randomly generated sensor to any anchor node. This means the four anchor nodes are placed some distance away from the boundary of the monitored area.

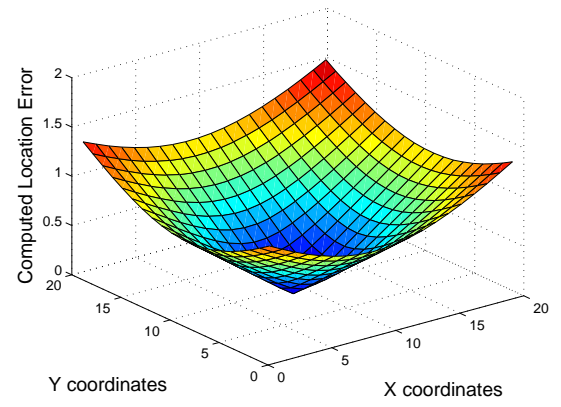

Figure 7: Position errors vs. real positions when $\lambda=0.1$ in the $x-y$ plane with $z=5$.

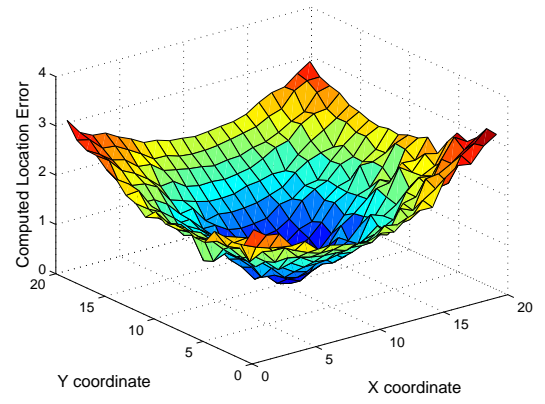

Figure 8: Position errors vs. real positions when $\lambda=2.5$ in the $x-y$ plane with $z=5$.

Next we consider the scenario when sensors are randomly deployed in a cubic space with lower-left corner $(1,1,1)$ and upper-right corner $(19,19,19)$. The four anchor nodes are still located at $(0,0,0),(20,0,0),(0,20,0)$ and $(0,0,20)$, respectively. For each $\lambda$ value, we try 2000 random sensor positions. The averaged results are reported in Fig. 9. Note that in this study, $I$ is selected from $\{1,2,3,4\}$ in order to demonstrate the effectiveness of UPS when applied to positioning mobile underwater vehicles.

We obtain three observations from Fig. 9. First, as $I$ increases, position error decreases. This is because averaging over larger number of beacon intervals to compute $k_{1} k_{2}$ and $k_{3}$ can better smooth out the effects of measuring errors in $\Delta t_{1}^{i}, \Delta t_{2}^{i}$ and $\Delta t_{3}^{i}$, thus produce improved result. A detailed theoretical explanation comes from Subsection 4.2. As $I$ increases, $\sigma_{1}^{2}, \sigma_{2}^{2}$ and $\sigma_{3}^{2}$ will decrease, and thus $V(x), V(y)$ and $V(z)$ will decrease. Then the errors from estimating the coordinates of sensors by $x, y$ and $z$ will decrease, implying that the position error will become smaller. Second, position error increases as $\lambda$ increases. This is reasonable in the underwater acoustic channel, in which a higher $\lambda$ comes from an even higher transmission rate when asymmetry commonly exists between the transmitter and the receiver. Such characteristic of the underwater medium brings significant multipath interference 
fact, if $\lambda$ increases which means $v$ increases at an even larger pace, $v^{2} / \lambda^{2}$ increases. As a result, $V(x), V(y)$ and $V(z)$ increase so that the errors from estimating the coordinates of the sensor by $x$ and $y$ and $z$ increase. Thus the larger the arrival rate, the larger the position error. Third, in the situation of small $\lambda$, for example $\lambda \approx 0.5$ as shown in Fig. 9, the location errors vary very little with the number of beacon intervals $I$. When $\lambda$ is relatively high, $I$ plays a more important role. The higher the $\lambda$, the bigger the difference induced from $I$. This observation is analogous to the terrestrial wireless communication channels, in which coherenct time is introduced to depict a period of time where the channel behavior or model can be considered as stationary. For underwater wireless communications, not only temporal coherence but also spatial and frequency coherences [18] are significant parameters for signal propagation through acoustic channels with multiple paths. Based on the third observation, $\lambda$ in underwater communications should not be neglected in estimating the coherence parameters. Note that rotating the square-cube monitored space within the open space formed by anchor nodes A, B, C and D, we obtain very similar results.

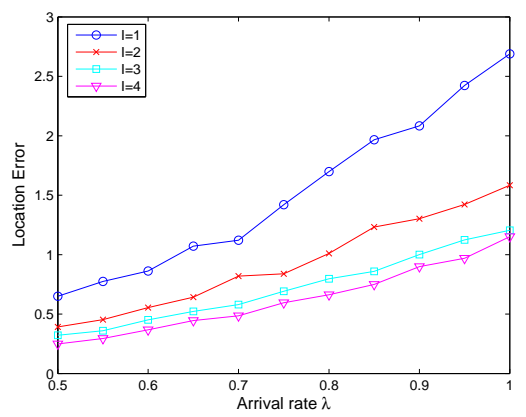

Figure 9: Position errors vs. $\lambda$ where $\angle B A C=90^{\circ}$.

In the following, we report the simulation results when $\angle B A C \leq 90^{\circ}$. In this simulation, the four anchor nodes are located at $(0,0,0),\left(X_{B}, Y_{B}, 0\right),\left(X_{C}, Y_{C}, 0\right)$, and $\left(0,0, Z_{D}\right)$, respectively, where $X_{B}, Y_{B}, X_{C}, Y_{C}$ and $Z_{D}$ are randomly drawn from $[5,20]$. 2000 sensors are randomly placed within the overlapping space formed by the anchor nodes $(A, B, C, D)$ and the cube space with corners $(0,0,0)$ and $(20,20,20)$. Fig. 10 reports the position error $v s$. $\lambda$. Note that the observations from Fig. 10 is very similar to those from Fig. 9. Nevertheless, for the same $\lambda$, the acute angle case performs slightly better than the right angle one.

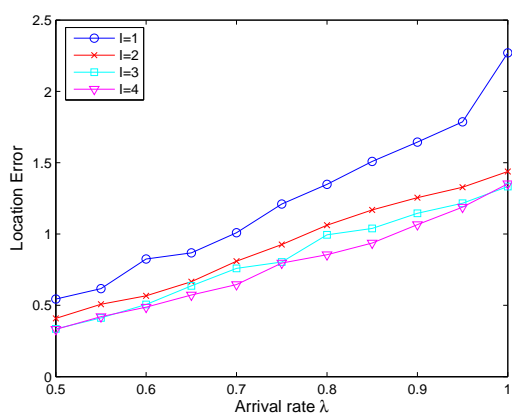

Figure 10: Position errors vs. $\lambda$ where $\angle B A C \leq 90^{\circ}$.

\section{Conclusion and Future Research}

In this paper, we propose UPS, a silent underwater positioning scheme for UWA-SNs. UPS is superior to existing systems in many aspects such as synchronization-free, low computation overhead, etc. To evaluate the performance of UPS, we model the underwater acoustic channel with a modified UWB S-V model, and conduct both theoretical analysis and simulation study. Our scheme is simple and effective. 
As a future research, we will study the impact of the channel modeling error on the position error of UPS. In addition, we intend to design a general framework based on projection such that localization algorithms proposed for 2D terrestrial sensor networks can be easily tailored to 3D underwater acoustic sensor networks. By this approach, the anchor node at the seabed is expected to be saved. 


\section{References}

[1] Global Positioning System standard - Positioning Service Specification, 2nd Edition, June 2, 1995.

[2] MIT Books On Line, Square Roots by Newton's Method, http://wwwmitpress.mit.edu/sicp/chapter1/node9.html.

[3] Underwater Acoustics Technical Guides - Speed of Sound in Seawater, http://www.npl.co.uk/accoustics/techguides/soundseawater/speedsw.pdf.

[4] Di Benedetto and Giancola, Understanding Ultra Wide Band Radio Fundamentals, Prentice Hall Communications Engineering and Emerging Technology, ISBN: 0131480030, Prentice Hall 2004.

[5] T.S. Rappaport, Wireless Communications: Principles and Practice, 2nd Edition, Prentice Hall, 2002.

[6] I. F. Akyildiz, D. Pompili, T. Melodia, "Underwater Acoustic Sensor Networks: Research Challenges," Elsevier's Journal of Ad Hoc Networks, Vol. 3, Issue 3, 2005, pp. 257-279.

[7] T. C. Austin, R. P. Stokey, and K. M. Sharp, PARADIGM: A Buoy-Based System for AUV Navigation and Tracking, in Proceedings of MTS/IEEE Oceans, 2000.

[8] C. Bechaz and H. Thomas, GIB system: The underwater GPS solution, in Proceedings of 5th Europe Conference on Underwater Acoustics, May 2000.

[9] N. Bulusu, J. Heidemann and D. Estrin, GPS-less Low Cost Outdoor Localization for Very Small Devices, IEEE Personal Communications Magazine, Special Issue on Networking the Physical World, August 2000.

[10] J.J. Caffery, Jr and G. L. Stüber, Overview of Radiolocation in CDMA Cellular Systems, IEEE Communications Magazine, pp. 38-45, 1998.

[11] J.J. Caffery, Jr and G. L. Stüber, Subscriber Location in CDMA Cellular Networks, IEEE Transactions on Vehicular Technology, Vol. 47, No. 2, pp. 406-416, May 1998.

[12] J.A. Catipovic, A.B. Baggeroer, K.Von Der Heydt and D. Koelsch, Design and Performance Analysis of a Digital Telemetry System for Short Range Underwater Channel, IEEE Journal of Oceanic Engineering, Vol. OE-9, No. 4, pp.242-252, Oct. 1984

[13] V. Chandrasekhar, W. K.G. Seah, Area Localization Scheme for Underwater Sensor Networks, Proceedings of the IEEE OCEANS Asia Pacific Conference, May 16-19, 2006.

[14] V. Chandrasekhar, W. K.G. Seah, Y. S. Choo, and H. V. Ee, Localization in Underwater Sensor Networks Survey and Challenges, Proceedings of ACM WUWNet, pp. 33-40, 2006.

[15] X. Cheng, A. Thaeler, G. Xue and D. Chen TPS: A Time-Based Positioning Scheme for Outdoor Wireless Sensor Networks, IEEE INFOCOM 2004, Vol. 4, pp. 2685-2696, 2004

[16] X. Geng and A. Zielinski, An Eigenpath Underwater Acoustic Communication Channel Model, OCEANS '95. MTS/IEEE. 'Challenges of Our Changing Global Environment'. Conference Proceedings, Vol 2, Oct.1995 pp:1189 - 1196

[17] M. Hahn and J. Rice, Undersea Navigation via a Distributed Acoustic Communicaiton Network, Proceedings of the Turkish International Conference on Acoustics, July 4-8, 2005.

[18] W. Jobst and X. Zabalgogeazcoa, Coherence Estimates for Signals Propagating through Acoustic Channels with Multiple Paths, Journal of the Acoustic Society of America, Vol.65, No.3, pp. 622-630, Mar. 1979

[19] F. Koushanfar, S. Slijepcevic, M. Potkonjak, and A. Sangiovanni-Vincentelli, Location Discovery in Ad-Hoc Wireless Sensor Networks, in Ad Hoc Wireless Networking (editors X. Cheng, X. Huang and D.-Z. Du), pp. 137-173, 2003. 
[20] Q. Liang, S. W. Samn, and X. Cheng, Outdoor UWB Channel Modeling in Rich Scattering and Time-Varying Environment, manuscript.

[21] F. Liu, X. Cheng, D. Hua, and D. Chen, Range-Difference Based Location Discovery for Sensor Networks with Short Range Beacons, to appear in International Journal on Ad Hoc and Ubiquitous Computing.

[22] A. Nasipuri and K. Li, A Directionality Based Location Discovery Scheme for Wireless Sensor Networks, WSNA'02, pp. 105-111, 2002.

[23] D. Niculescu, and B. Nath, Ad-Hoc Positioning System (APS), IEEE GlobeCom, 2001.

[24] J. Partan, J. Kurose and B.N. Levine, A Survey of Practical Issues in Underwater Networks, Proceedings of the 1st ACM international workshop on Underwater networks, International Conference on Mobile Computing and Networking, pp. 17-24, Sep. 2006, Los Angeles, CA.

[25] G.H. Sandsmark and A. Solstad, Adaptive Beam Forming and Adaptive Equalization for High-Speed Underwater Acoustic Data Transmission, Proc. of Underwater Defence Tech. Conference, pp. 707-712, Apr. 1991

[26] A. Syed and J. Heidemann, Time Synchronization for High Latency Acoustic Networks, IEEE INFOCOM 2006.

[27] A. Thaeler, M. Ding, and X. Cheng, iTPS: An Improved Location Discovery Scheme for Sensor Networks with Long Range Beacons, Journal of Parallel and Distributed Computing, Vol. 65, No. 2, pp. 98-106, February 2005.

[28] Y. Zhang and L. Cheng, A distributed protocol for multi-hop underwater robot positioning, in Proceedings of IEEE International Conference on Robotics and Biomimetics, pages 480484, Aug 2004.

[29] Z. Zhou, J.-H. Cui, and S. Zhou, Localization for Large-scale Underwater Sensor Networks, UCONN CSE Technical report: UbiNet-TR06-04, December 2006. 